\title{
The influence of fish consumption on polyunsaturated fatty acid status
}

\author{
M.C. Conway ${ }^{1}$, E.M. McSorley ${ }^{1}$, M.S. Mulhern ${ }^{1}$, J.J. Strain ${ }^{1}$, K. Broberg ${ }^{2}$, \\ E. van Wijngaarden ${ }^{3}$ and A.J. Yeates ${ }^{1}$ \\ ${ }^{1}$ Nutrition Innovation Centre for Food and Health, Ulster University Coleraine, Cromore Road, Coleraine, Northern \\ Ireland BT52 1SA, ${ }^{2}$ Institute of Environmental Medicine, Karolinska Institutet, Stockholm, Sweden and ${ }^{3}$ University of \\ Rochester, School of Medicine and Dentistry, United States.
}

Polyunsaturated fatty acids (PUFA) have an important role in immune function ${ }^{(1)}$, cardiovascular health $^{(2)}$ and foetal development $^{(3)}$. Fish consumption is a major determinant of improved long chain (LC) n-3 PUFA status ${ }^{(4)}$. Genetic variation in the fatty acid desaturase (FADS) genotype also has an influence on PUFA status ${ }^{(5)}$. The aim of the current study was to investigate whether fish consumption influences PUFA status in women of childbearing age.

Female participants of childbearing age $(n=49)$ were recruited to take part in an 8 -week intervention trial. Participants provided a buccal swab, which was analysed for FADS genotype, on which participants were stratified (SNP rs3834458) and randomised to consume zero (control), one, or two portions (140 g/portion) of oily fish (tuna or sardines) per week. Blood samples were taken pre- and post-intervention and analysed for serum total PUFA status using GC-MS. Statistical analysis was completed using IBM SPSS Statistics v24.

The median (IQR) age of participants was $23(20,30)$ years. ANCOVA with post-hoc comparison was used to assess the effect of intervention on PUFA status (Table 1). Participants consuming two portions of fish per week had significantly higher concentrations of eicosapentaenoic acid (EPA), docosahexaenoic acid (DHA) and total n-3 PUFA than those consuming one portion or zero portions. The n-6:n-3 ratio was also significantly influenced by fish intake with consumption of two portions of fish per week resulting in a significantly lower $n-6: n-3$ ratio when compared to the other intervention groups. The median (IQR) n-6:n-3 ratio for those consuming 2 portions of fish per week was lower for those with the homozygous TT genotype (5.394 (4.822, 6.026) compared to those with genetic variation of Tdel, or del genotype (6.617 (4.720, 7.085).

Table 1. The effect of intervention on PUFA status $(\mathrm{mg} / \mathrm{ml})$ at post intervention expressed as Median (IQR)

\begin{tabular}{|c|c|c|c|c|c|}
\hline \multirow[b]{2}{*}{ PUFA } & \multicolumn{3}{|c|}{ Median (IQR) } & \multirow[b]{2}{*}{$P$} & \multirow[b]{2}{*}{ Partial eta squared } \\
\hline & Control $(n=18)$ & 1 portion $(n=14)$ & 2 portions $(n=17)$ & & \\
\hline LA & $0.275(0.229,0.301)$ & $0.260(0.245,0.300)$ & $0.291(0.255,0.322)$ & 0.597 & $0 \cdot 026$ \\
\hline ALA & $0.013(0.012,0.014)$ & $0.012(0.012,0.0130$ & $0.013(0.012,0.016)$ & 0.912 & 0.005 \\
\hline $\mathbf{A A}$ & $0.064(0.055,0.075)$ & $0.068(0.055,0.083)$ & $0.069(0.059,0.078)$ & $0 \cdot 532$ & $0 \cdot 032$ \\
\hline EPA & $0.013(0.012,0.013)^{\mathrm{a}}$ & $0.014(0.013,0.015)^{\mathrm{a}}$ & $0.015(0.013,0.025)^{\mathrm{b}}$ & 0.001 & $0 \cdot 327$ \\
\hline DHA & $0.021(0.016,0.023)^{\mathrm{a}}$ & $0.022(0.020,0.027)^{\mathrm{a}}$ & $0.028(0.024,0.034)^{\mathrm{b}}$ & $<0.001$ & $0 \cdot 346$ \\
\hline Total n-6 & $0.347(0.294,0.364)$ & $0.327(0.312,0.376)$ & $0.367(0 \cdot 316,0 \cdot 392)$ & 0.640 & 0.023 \\
\hline Total n-3 & $0.045(0.041,0.052)^{\mathrm{a}}$ & $0.046(0.037,0.054)^{\mathrm{a}}$ & $0.059(0.051,0.070)^{\mathrm{b}}$ & $<0.001$ & $0 \cdot 359$ \\
\hline n6:n3 ratio & $7.293(6.860,8.173)^{a}$ & $7.340(6.699,7.855)^{\mathrm{a}}$ & $6.026(4.809,6.969)^{\mathrm{b}}$ & 0.001 & $0 \cdot 289$ \\
\hline
\end{tabular}

LA, linoleic acid; ALA, alpha-linolenic acid; AA, arachidonic acid; EPA, eicosapentaenoic acid; DHA, docosahexaenoic acid; all variables log transformed, $P$ value is for between intervention group change from baseline (ANCOVA, adjusting for age, weight, BMI and baseline PUFA); different letters represent significant difference from each other $p<0 \cdot 05$; IQR expressed as $25^{\text {th }}, 75^{\text {th }}$ percentile

In conclusion, consumption of two portions of fish per week was shown to have beneficial effects on n-3 PUFA status and the n-6: n-3 ratio. The n-6:n-3 ratio was lower in those with the TT compared to those with Tdel or del genotype, indicating genetic variation may also influence PUFA status.

1. Calder (2015) J Parenter Enteral Nutr 39, (1 Suppl): 18S-32S.

2. Manuelli et al. (2017) Int $J$ Mol Sci 18(7).

3. Coletta et al. (2010) Rev Obstet Gynecol 3(4), 163-171.

4. Weichselbaum E et al. (2013) Nutr Bull 38, 128-177.

5. Minihane (2016) Nutrients 8, 123. 